\title{
Alginate dressings for treating pressure ulcers (Protocol)
}

\author{
Dumville JC, Keogh SJ, Stubbs N, Walker RM
}

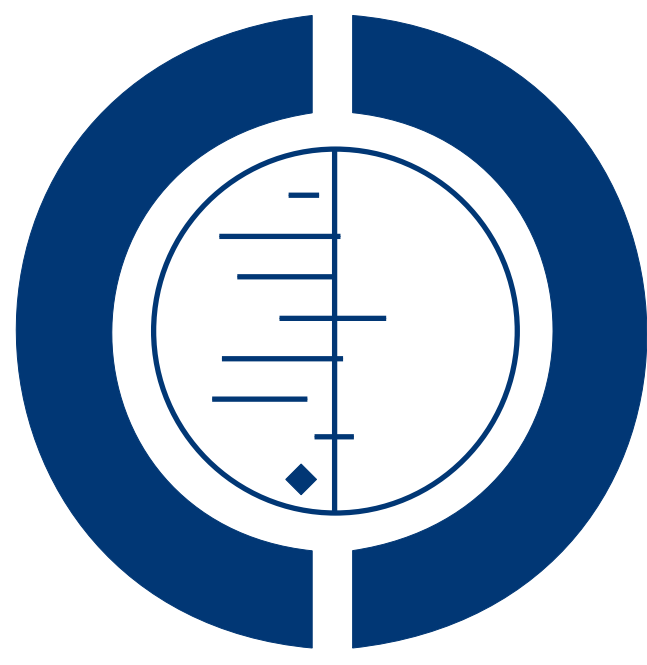

\section{THE COCHRANE COLLABORATION $^{\circledR}$}

This is a reprint of a Cochrane protocol, prepared and maintained by The Cochrane Collaboration and published in The Cochrane Library 2014, Issue 8

http://www.thecochranelibrary.com

\section{WILEY}


TABLE OF CONTENTS

HEADER . . . . . . . . . . . . . . . . . . . . . . . . . . . . . . . . . . . . 1

ABSTRACT . . . . . . . . . . . . . . . . . . . . . . . . . . . . . . . . . . . . . . . . .

BACKGROUND . . . . . . . . . . . . . . . . . . . . . . . . . . . . . . . . . . . .

OBJECTIVES . . . . . . . . . . . . . . . . . . . . . . . . . . . . . . . . . . . . . . . . . .

METHODS . . . . . . . . . . . . . . . . . . . . . . . . . . . . . . . . . . . . . . 44

ACKNOWLEDGEMENTS . . . . . . . . . . . . . . . . . . . . . . . . . . . . . . . . . . . . . . . . . .

REFERENCES . . . . . . . . . . . . . . . . . . . . . . . . . . . . . . . . . . . . . . . . .

APPENDICES . . . . . . . . . . . . . . . . . . . . . . . . . . . . . . . . . . . . . 11

CONTRIBUTIONS OF AUTHORS . . . . . . . . . . . . . . . . . . . . . . . . . . . . . . . . . . . . . . .

DECLARATIONS OF INTEREST . . . . . . . . . . . . . . . . . . . . . . . . . . . . . . . . 14

SOURCES OF SUPPORT . . . . . . . . . . . . . . . . . . . . . . . . . . . . . . . . . . 


\title{
[Intervention Protocol]
}

\section{Alginate dressings for treating pressure ulcers}

\author{
Jo C Dumville ${ }^{1}$, Samantha J Keogh ${ }^{2}$, Nikki Stubbs ${ }^{3}$, Rachel M Walker ${ }^{2}$ \\ ${ }^{1}$ School of Nursing, Midwifery and Social Work, University of Manchester, Manchester, UK. ${ }^{2}$ NHMRC Centre of Research Excellence \\ in Nursing, Centre for Health Practice Innovation, Griffith Health Institute, Griffith University, Brisbane, Australia. ${ }^{3}$ Wound Prevention \\ and Management Service, Leeds Community Healthcare NHS Trust, St Mary's Hospital, Leeds, UK
}

Contact address: Jo C Dumville, School of Nursing, Midwifery and Social Work, University of Manchester, Manchester, M13 9PL, UK. jo.dumville@manchester.ac.uk.

Editorial group: Cochrane Wounds Group.

Publication status and date: New, published in Issue 8, 2014.

Citation: Dumville JC, Keogh SJ, Stubbs N, Walker RM. Alginate dressings for treating pressure ulcers. Cochrane Database of Systematic Reviews 2014, Issue 8. Art. No.: CD011277. DOI: 10.1002/14651858.CD011277.

Copyright (C) 2014 The Cochrane Collaboration. Published by John Wiley \& Sons, Ltd.

\begin{abstract}
A B S T R A C T
This is the protocol for a review and there is no abstract. The objectives are as follows:

To assess the effects of alginate dressings for treating pressure ulcers in any care setting.
\end{abstract}

\section{B A C K G R O U N D}

\section{Description of the condition}

Pressure ulcers, also known as bedsores, decubitus ulcers and pressure injuries, are localised areas of injury to the skin or the underlying tissue, or both. They often occur in areas with a bony prominence such as the sacrum (base of the spine) and the heel (Vanderwee 2007) and are caused by external forces such as pressure, or shear, or a combination of both (EPUAP-NPUAP 2009). Populations at risk of pressure ulceration include those with spinal cord injuries (Gefen 2014) and those immobilised or with limited mobility, such as elderly people and people with acute or chronic conditions that might limit movement or bodily sensation, or both (Allman 1997; Bergstrom 1998; Berlowitz 1990; Berlowitz 1997; Brandeis 1994). Incontinence can also increase risk of ulceration by producing a detrimental environment for the skin (Brandeis 1994). Impaired nutritional status may increase risk as well (Allman 1997; Donini 2005); however, evidence for the effectiveness of nutritional intake interventions for preventing or treating pressure ulcers is currently limited (Langer 2003; Smith 2013).

Mobility produces relief from pressure within the body through regular, often subconscious, shifts in position when sitting or lying. These movements, triggered by a reduction in oxygen levels at pressure points and possible discomfort, distribute pressure from contact at the surface, thus reducing the compression of soft tissue against bone (Gebhardt 2002). Populations with limited autonomous movement or conditions that dull body sensation, or both (as described above), are at risk of failing to achieve adequate pressure relief. Prolonged exposure of an area of the body to pressure or compression can interrupt the local blood circulation and trigger a cascade of biochemical changes that may lead to tissue damage and ulceration. Immobility can also lead to increased damage from shear and friction, for example, when people are pulled into position in chairs and beds.

Pressure ulcers vary in severity. One of the most widely recognised systems for categorising pressure ulcers is that of the National Pressure Ulcer Advisory Panel, which is summarised below (NPUAP 2009). 
- Category/Stage I-non-blanchable erythema: "Intact skin with non-blanchable redness of a localised area usually over a bony prominence. Darkly pigmented skin may not have visible blanching; its colour may differ from the surrounding area. The area may be painful, firm, soft, warmer or cooler as compared to adjacent tissue. Category I may be difficult to detect in individuals with dark skin tones. May indicate 'at risk' persons."

- Category/Stage II-partial thickness: "Partial thickness loss of dermis presenting as a shallow open ulcer with a red pink wound bed, without slough. May also present as an intact or open/ruptured serum-filled or sero-sanguinous filled blister. Presents as a shiny or dry shallow ulcer without slough or bruising (bruising indicates deep tissue injury). This category should not be used to describe skin tears, tape burns, incontinence associated dermatitis, maceration or excoriation."

- Category/Stage III-full thickness skin loss: "Full thickness tissue loss. Subcutaneous fat may be visible but bone, tendon or muscle are not exposed. Slough may be present but does not obscure the depth of tissue loss. May include undermining and tunnelling. The depth of a Category/Stage III pressure ulcer varies by anatomical location. The bridge of the nose, ear, occiput and malleolus do not have (adipose) subcutaneous tissue and Category/Stage III ulcers can be shallow. In contrast, areas of significant adiposity can develop extremely deep Category/Stage III pressure ulcers. Bone/tendon is not visible or directly palpable."

- Category/Stage IV-full thickness tissue loss: "Full thickness tissue loss with exposed bone, tendon or muscle. Slough or eschar may be present. Often includes undermining and tunnelling. The depth of a Category/Stage IV pressure ulcer varies by anatomical location. The bridge of the nose, ear, occiput and malleolus do not have (adipose) subcutaneous tissue and these ulcers can be shallow. Category/Stage IV ulcers can extend into muscle and/or supporting structures (e.g., fascia, tendon or joint capsule) making osteomyelitis or osteitis likely to occur. Exposed bone/muscle is visible or directly palpable."

Pressure ulcers are relatively common, but complex, wounds. Prevalence estimates vary according to the population assessed, the data collection methods used and decisions made about whether Stage I pressure ulcers should be included (as there is no active wound at this stage, but patients are 'at risk'). A large survey of hospital patients undertaken in several European countries returned a pressure ulcer prevalence (Stage II and above) of $10.5 \%$ (Vanderwee 2007). In 2009, a US estimate for pressure ulcer prevalence (Stage II and above) across acute-care, long-term care and rehabilitation settings was $9.0 \%$, with prevalence highest in longterm acute-care settings (26\%) (VanGilder 2009). In the UK, national pressure ulcer data are collected across community and acute settings-although data collection is not yet universal-as part of the National Health Service (NHS) Safety Thermometer initiative (Power 2012). Five per cent of patients across these settings were estimated to have a pressure ulcer in January 2014 (National
Safety Thermometer Data 2014).

We note that all prevalence figures quoted above are for populations currently receiving medical care. The point prevalence of pressure ulceration in the total adult population was recently estimated using a cross-sectional survey undertaken in Leeds, UK. Of the total adult population of 751,485 , the point prevalence of pressure ulceration per 1000 was 0.31 (Hall, personal communication). UK pressure ulcer prevalence estimates specifically for community settings have reported rates of 0.77 per 1000 adults in a UK urban area (Stevenson 2013).

Pressure ulcers have a large impact on those affected; the ulcers can be painful and may become seriously infected or malodorous. It has been shown that-after adjustment for age, sex and co-morbiditiespeople with pressure ulcers have lower health-related quality of life than those without pressure ulcers (Essex 2009). The financial cost of treating ulcers in the UK was recently estimated as between GBP 1214 for a Stage I ulcer and GBP 14,108 for a Stage IV ulcer (Dealey 2012). In 2004 the total annual cost of treating pressure ulcers in the UK was estimated as GBP 1.4 to 2.1 billion, which was equivalent to $4 \%$ of the total NHS expenditure (Bennett 2004). Pressure ulcers have been shown to increase length of hospital stay and associated hospital costs (Allman 1999). Figures from the USA suggest that for half a million hospital stays in 2006, 'pressure ulcer' was noted as a diagnosis; for adults, the total hospital cost for these stays was USD 11 billion (Russo 2008). Costs to the Australian healthcare system for treating pressure ulceration have been estimated at AUD 285 million per annum (Graves 2005).

\section{Description of the intervention}

Two main strategies are used in the treatment of pressure ulcers, namely, relief of pressure-commonly using specialist support surfaces (McInnes 2011)-alongside management of the wound environment using wound dressings. Other general strategies include providing patient education, managing pain, optimising circulation/perfusion, optimising nutrition, performing surgical wound closure and treating clinical infection (AWMA 2012; EPUAP-NPUAP 2009).

Dressings are widely used in wound care, with the aim of protecting the wound while promoting healing. Classification of dressings usually depends on the key material used in their construction. Several attributes of an ideal wound dressing have been described (e.g. BNF 2013), including:

- the ability of the dressing to absorb and contain exudate without leakage or strike-through;

- lack of particulate contaminants left in the wound by the dressing;

- thermal insulation;

- permeability to water but not to bacteria;

- avoidance of wound trauma on dressing removal;

- frequency with which the dressing needs to be changed;

- provision of pain relief; and 
- comfort.

Alginate dressings are the focus of this review; their properties are described below. As alginate dressings are likely to be evaluated against one of the many wound dressings available, a description of potential comparators, based on the British National Formulary structure (BNF 2013), is also provided. Dressings are listed below by their generic names and, when possible, with examples of corresponding trade names and manufacturers. Dressing names, manufacturers and distributors may vary between countries.

\section{Basic wound contact dressings}

- Low-adherence dressings and wound contact materials: These are usually cotton pads that are placed in direct contact with the wound. Examples include paraffin gauze dressing, BP 1993 and Xeroform (Covidien) dressing-a non-adherent petrolatum blend with $3 \%$ bismuth tribromophenate on fine mesh gauze.

- Absorbent dressings: These can be applied directly to the wound or used as secondary absorbent layers in the management of heavily exuding wounds. Examples include Primapore (Smith $\&$ Nephew), Mepore (Mölnlycke) and absorbent cotton gauze (BP 1988).

\section{Advanced wound dressings}

- Alginate dressings: These are highly absorbent and come in the form of calcium alginate or calcium sodium alginate, and they can be combined with collagen. The alginate forms a gel when in contact with the wound surface, which can be lifted off at dressing removal or rinsed away with sterile saline. Bonding to a secondary viscose pad increases absorbency. Examples include Curasorb (Covidien), SeaSorb (Coloplast) and Sorbsan (Unomedical).

- Foam dressings: These dressings normally contain hydrophilic polyurethane foam and are designed to absorb wound exudate and maintain a moist wound surface. They are produced in a variety of versions: Some foam dressings include additional absorbent materials, such as viscose and acrylate fibres or particles of superabsorbent polyacrylate; others are siliconecoated for non-traumatic removal. Examples include Allevyn (Smith \& Nephew), Biatain (Coloplast) and Tegaderm (3M).

- Hydrogel dressings: These consist of cross-linked insoluble polymers (i.e. starch or carboxymethylcellulose) and up to $96 \%$ water. They are designed to absorb wound exudate or to rehydrate a wound, depending on wound moisture levels. They are supplied as flat sheets, as an amorphous hydrogel or as beads. Examples include ActiformCool (Activa) and Aquaflo (Covidien).

- Films-permeable film and membrane dressings: These dressings are permeable to water vapour and oxygen but not to water or micro-organisms. Examples includeTegaderm (3M) and Opsite (Smith \& Nephew).

- Soft polymer dressings: These dressings are moderately absorbent and are composed of a soft silicone polymer held in a non-adherent layer. Examples include Mepitel (Mölnlycke) and Urgotul (Urgo).

- Hydrocolloid dressings: These occlusive dressings are usually composed of a hydrocolloid matrix bonded onto a vapour-permeable film or foam backing. This matrix forms a gel that provides a moist environment when in contact with the wound surface. Examples include GranuFLEX (ConvaTec) and NU DERM (Systagenix). Fibrous alternatives have been developed that resemble alginates, are not occlusive and are more absorbent than standard hydrocolloid dressings. An example is Aquacel (ConvaTec).

- Capillary action dressings: These consist of an absorbent core of hydrophilic fibres held between two low-adherent contact layers. Examples include Advadraw (Advancis) and Vacutx (Protex).

- Odour absorbent dressings: These dressings contain charcoal and are used to absorb wound odour, often in conjunction with a secondary dressing to improve absorbency. An example is CarboFLEX (ConvaTec).

\section{Antimicrobial dressings}

- Honey-impregnated dressings: These dressings contain medical-grade honey, which is thought to have antimicrobial and anti-inflammatory properties and can be used for acute or chronic wounds. Examples include Medihoney (Medihoney) and Activon Tulle (Advancis).

- Iodine-impregnated dressings: These dressings release free iodine, which is thought to act as a wound antiseptic, when exposed to wound exudate. Examples include Iodoflex (Smith \& Nephew) and Iodozyme (Insense).

- Silver-impregnated dressings: These dressings are used to treat infected wounds, as silver ions are thought to have antimicrobial properties. Silver versions of most dressing types are available (e.g. silver foam, silver hydrocolloid). Examples include Acticoat (Smith \& Nephew) and Urgosorb Silver (Urgo).

- Other antimicrobial dressings: These dressings are composed of a gauze or low-adherent dressing impregnated with an ointment thought to have antimicrobial properties. Examples include chlorhexidine gauze dressing (Smith \& Nephew) and Cutimed Sorbact (BSN Medical).

\section{Specialist dressings}

- Protease-modulating matrix dressings: These dressings alter the activity of proteolytic enzymes in chronic wounds. Examples include Promogran (Systagenix) and Sorbion (H \& R). 
The diversity of dressings available to health professionals (including variations within each type) can make evidence-informed decision making challenging. Furthermore, whilst dressings may be viewed as 'inert' and cheap products, increasingly they are being formulated with an 'active' ingredient (e.g. silver) or with other antimicrobial products. With increasingly sophisticated technology applied to wound care, practitioners need to know how effective these-often expensive-dressings are compared with more traditional, usually less costly, options. Data on the current use of dressings for the treatment of pressure ulcers are limited, although older studies have shown wide variation in practice and wound (wound type) care knowledge (Pieper 1995).

\section{How the intervention might work}

Animal experiments conducted over 40 years ago suggest that acute wounds heal more quickly when their surfaces are kept moist rather than left to dry and scab (Winter 1962; Winter 1963a; Winter 1963b). A moist environment is thought to provide optimal conditions for the cells involved in the healing process, as well as allowing autolytic debridement (removal of dead tissue by natural processes), which is thought to be an important part of the healing pathway (Cardinal 2009). The desire to maintain a moist wound environment is a key driver for the use of wound dressings. Wound dressings vary in their level of absorbency, so that a very wet wound can be treated with an absorbent dressing (such as a foam dressing) to draw excess moisture away and avoid skin damage, whilst a drier wound can be treated with a more occlusive dressing to maintain a moist environment. Alginate dressings contain sodium, or sodium and calcium, salts of alginic acid. These alginate salts are highly hydrophilic and can absorb large volumes of wound exudate.

\section{Why it is important to do this review}

Pressure ulcers are a relatively common complex type of wound that have a negative impact on people's lives and incur high costs for health services. Dressings are widely used as treatment for pressure ulcers, and understanding the existing evidence base and potential uncertainty around the clinical efficacy and cost-effectiveness of different dressing types is important for decision making in this area.

A key international guideline recommends that a dressing should be chosen "that keeps the wound bed moist"; this recommendation was classed as level $\mathrm{C}$ evidence that is "supported by indirect evidence (e.g., studies in normal human subjects, humans with other types of chronic wounds, animal models) and/or expert opinion" (EPUAP-NPUAP 2009). The same guidelines suggest that alginate dressings are used to treat pressure ulcers in various scenarios, mainly for the treatment of moderately or heavily ex- uding ulcers, but these recommendations are based on limited evidence (EPUAP-NPUAP 2009).

Two notable systematic reviews of treatment for pressure ulcers have included trials of dressings (Reddy 2008; Smith 2013). Reddy 2008 included five trials of alginates in people with pressure ulcers. These studies were included as part of a much larger review that assessed multiple interventions for treating pressure ulcers. The report stated, "No single dressing was consistently superior to other dressings in the trials of pressure ulcers we examined"; however, because of the breadth of the review, detailed examination of effect estimates and quantification of uncertainty around the alginate trials were difficult. One included study reported that a calcium alginate dressing showed statistically significant improvement in pressure ulcer healing compared with dextranomer paste (with healing measured as mean wound surface area reduction per week). The search for trials for this review was done almost six years ago. The more recent review seems to include dressing interventions but does not mention alginates specifically (Smith 2013). We conclude that up-to-date and transparent information on evidence for the use of alginate dressings to treat pressure ulcers is required.

This review is part of a suite of Cochrane reviews investigating the use of dressings in the treatment of pressure ulcers . Each review will focus on a particular dressing type. These reviews will be summarised in an overview of reviews that will draw together all existing Cochrane review evidence regarding the use of dressings to treat pressure ulcers.

\section{O B J E C T I VES}

To assess the effects of alginate dressings for treating pressure ulcers in any care setting.

\section{METHODS}

\section{Criteria for considering studies for this review}

\section{Types of studies}

We will include published and unpublished randomised controlled trials (RCTs), including cluster RCTs (which also include studies in which multiple wounds on the same participant are treated with the allocated treatment and outcome data are collected and analysed for each wound), irrespective of language of report. RCTs reported only as abstracts will be included only when available data are sufficient for reasonable data extraction either from the abstract itself or from the study authors. Cross-over trials will be included only if outcome data are available from the end of 
the first treatment period before cross-over. Studies using quasirandomisation will be excluded.

\section{Types of participants}

We will include studies that recruited adults with a diagnosis of pressure ulcer (Stage II or above) managed in any care setting. We will exclude participants with Stage I ulcers. We will accept study authors' definitions of what they classed as Stage II or above, unless it is clear that they included wounds with unbroken skin. Studies that recruited participants with Stage II or higher pressure ulcers alongside people with other types of chronic wounds (e.g. leg or foot ulcers, or both) will be included if the results for people with relevant pressure ulcers are presented separately (or are available from the study authors). Similarly, when a trial included both Stage I and more advanced stages of pressure ulcers, it will be included in the review only if data on ulcers of Stage II and above are reported separately or are available on request from study authors.

\section{Types of interventions}

The primary intervention will be alginate wound dressings (BNF 2013). We will include any RCT in which the use of a specific alginate dressing is the only systematic difference between treatment groups. We anticipate that probable comparisons will include different types of alginate dressings compared with each other; alginate dressings compared with other dressing types; and alginate dressings compared with other interventions (possibly non-dressing treatments, e.g. topical treatments).

\section{Types of outcome measures}

\section{Primary outcomes}

The primary outcome for this review is complete wound healing. We note that, because wound healing is a subjective outcome, it can be at high risk of measurement bias when outcome assessment is not blinded. For this review, we will regard the following as providing the most relevant and rigorous measures of outcome.

- Time to complete wound healing (correctly analysed using censored data and preferably adjusted for prognostic co-variates such as baseline size). We will consider mean or median time to healing without survival analysis as a valid outcome only if reports specify that all wounds healed (i.e. if the trial authors regarded time to healing as a continuous measure, as there is no censoring).

- Proportion of ulcers healed during follow-up (frequency of complete healing).

When both time to healing and proportion of ulcers healed are reported, we will present all data in a summary outcome table for reference purposes, but we will focus on reporting the 'best' healing outcome available. We consider time to healing to be the best outcome. We anticipate presenting data for the latest time point available-unless an earlier time point is clearly the primary focus of the study, in which case data from multiple time points will be extracted. We will accept authors' definitions of what constituted a healed wound.

When time is analysed as a continuous measure, but it is not clear whether all wounds healed, or when change or rate of change in wound size is reported without adjustment for baseline size, we will document the use of the outcome in the study, but we will not extract, summarise or use the data in any meta-analysis.

\section{Secondary outcomes}

- Change (and rate of change) in wound size, with adjustment for baseline size (we will contact study authors to request adjusted means when not presented). When change or rate of change in wound size is reported without adjustment for baseline size, use of the outcome in the study will be documented, but data will not be extracted, summarised or used in any meta-analysis.

- Participant health-related quality of life/health status (measured using a standardised generic questionnaire such as EQ-5D (standardised instrument used to measure health outcomes), Short Form (SF)-36, SF-12 or SF-6 or woundspecific questionnaires such as the Cardiff Wound Impact Schedule). We will not include ad hoc measures of quality of life that are not likely to be validated and would not be common to multiple trials.

- Wound infection (when infection has been defined by the study authors).

- Other adverse events, including pain associated with the ulcer or experienced at dressing change (measured using survey/ questionnaire/data capture process or visual analogue scale), when a clear methodology for the collection of adverse event data was provided.

- Resource use (including measurements of resource use, such as number of dressing changes, number of nurse visits, length of hospital stay and whether reoperation/intervention was provided).

- Cost (allocated to resource use).

- Wound recurrence.

For all outcomes we will report outcome measures at the latest time point available (assumed to be length of follow-up if not specified) and the time point specified in the methods as being of primary interest (if this is different from latest time point available).

\section{Search methods for identification of studies}

\section{Electronic searches}


We will search the following electronic databases.

- The Cochrane Wounds Group Specialised Register.

- The Cochrane Central Register of Controlled Trials

(CENTRAL) (latest issue).

- The Database of Abstracts of Reviews of Effects (DARE)

(latest issue).

- The Health Technology Assessment Database (HTA) (latest issue).

- NHS Economic Evaluation Database latest issue).

- Ovid MEDLINE (1946 to date).

- Ovid MEDLINE (In-Process \& Other Non-Indexed Citations).

- Ovid EMBASE (1974 to date).

- EBSCO Cumulative Index to Nursing and Allied Health Literature (CINAHL) (1982 to date).

We will use the following provisional search strategy in the Cochrane Central Register of Controlled Trials (CENTRAL):

\#1 MeSH descriptor: [Occlusive Dressings] explode all trees \#2 MeSH descriptor: [Biological Dressings] explode all trees \#3 MeSH descriptor: [Alginates] explode all trees \#4 MeSH descriptor: [Hydrogels] explode all trees \#5 MeSH descriptor: [Silver] explode all trees \#6 MeSH descriptor: [Silver Sulfadiazine] explode all trees \#7 MeSH descriptor: [Honey] explode all trees \#8 MeSH descriptor: [Bandages, Hydrocolloid] explode all trees \#9 (dressing* or alginate* or hydrogel* or hydrocolloid* or "foam" or "bead" or "film" or "films" or tulle or gauze or non-adherent or "non adherent" or silver* or honey or matrix):ti,ab,kw $\# 10 \# 1$ or \#2 or \#3 or \#4 or \#5 or \#6 or \#7 or \#9 \#11 MeSH descriptor: [Pressure Ulcer] explode all trees \#12 (pressure next (ulcer* or sore* or injur*)):ti,ab,kw \#13 (decubitus next (ulcer* or sore*)):ti,ab,kw \#14 ((bed next sore*) or bedsore):ti,ab,kw

$\# 15$ \#11 or \#12 or \#13 or \#14

\#16 \#10 and \#15

We will combine the Ovid MEDLINE search with the Cochrane Highly Sensitive Search Strategy for identifying randomised trials in MEDLINE: sensitivity- and precision-maximising version (2008 revision) (Lefebvre 2011). We will combine the EMBASE search with the Ovid EMBASE filter developed by the UK Cochrane Centre (Lefebvre 2011). We will combine the CINAHL searches with the trial filters developed by the Scottish Intercollegiate Guidelines Network (SIGN 2011). We will apply no restrictions with respect to language, date of publication or study setting.

We will also search the following clinical trials registries.

- ClinicalTrials,gov (http://www.clinicaltrials.gov/).

- World Health Organization (WHO) International Clinical

Trials Registry Platform (http://apps.who.int/trialsearch/

Default.aspx).

- EU Clinical Trials Register (https:// www.clinicaltrialsregister.eu/).

\section{Searching other resources}

We will contact corresponding authors of trials and manufacturers and distributors of wound dressings. We will search the US Food and Drug Administration briefing documents used in the licensing of wound dressings. We will try to identify other potentially eligible trials or ancillary publications by searching the reference lists of retrieved included trials, as well as relevant systematic reviews, meta-analyses and health technology assessment reports.

\section{Data collection and analysis}

\section{Selection of studies}

Independently, two review authors will assess for relevance the titles and abstracts of the citations retrieved by the searches. After this initial assessment, we will obtain full-text copies of all studies believed to be potentially relevant. Independently, two review authors will check the full papers for eligibility; disagreements will be resolved by discussion, and, when required, the input of a third review author. When the eligibility of a study is unclear, we will attempt to contact study authors to ask for clarification. We will record all reasons for exclusion of studies for which we have obtained full copies. We will complete a PRISMA (Preferred Reporting Items for Systematic Reviews and Meta-Analyses) flow chart to summarise this process (Liberati 2009).

We will obtain all relevant publications when studies have been reported more than once. Whilst the study will be included only once in the review, all reports will be examined to ensure the maximal extraction of relevant data.

\section{Data extraction and management}

We will extract and summarise details of the eligible studies. Two review authors will extract data independently and will resolve disagreements by discussion, drawing on a third review author when required. When data are missing from reports, we will attempt to contact the study authors to obtain this information. When a study with more than two intervention arms is included, data will be extracted only from intervention and control groups that meet the eligibility criteria of the review.

We will extract the following data when possible on those trial arms that are relevant to the review.

- Country of origin.

- Type/grade/category of pressure ulcer.

- Location of pressure ulcer.

- Unit of randomisation and analysis (e.g. participant with single wound, participant with multiple wounds).

- Trial design (e.g. parallel, cluster).

- Care setting.

- Number of participants randomly assigned to each trial arm.

Alginate dressings for treating pressure ulcers (Protocol)

Copyright $\odot 2014$ The Cochrane Collaboration. Published by John Wiley \& Sons, Ltd. 
- Eligibility criteria and key baseline participant data.

- Details of treatment regimen received by each group.

- Duration of treatment.

- Details of any co-interventions provided.

- Primary and secondary outcome(s) (with definitions).

- Outcome data for primary and secondary outcomes (by group).

- Duration of follow-up.

- Number of withdrawals (by group).

- Publication status of study.

- Source of funding for trial.

\section{Assessment of risk of bias in included studies}

Independently, two review authors will assess the included studies that performed individual randomisation using the tool of The Cochrane Collaboration for assessing risk of bias (Higgins 2011a). This tool addresses six specific domains: sequence generation, allocation concealment, blinding, incomplete data, selective outcome reporting and other issues (Appendix 1). We will assess blinded outcome assessment and completeness of outcome data for each outcome separately. We will present our assessment of risk of bias using two 'Risk of bias' summary figures; one will provide a summary of bias for each item across all studies, and the second will provide a cross-tabulation of each trial using all risk of bias items. For trials using cluster randomisation, we will assess the risk of bias using the following domains: recruitment bias, baseline imbalance, loss of clusters, incorrect analysis and comparability with individually randomised trials (Higgins 2011b) (Appendix 2).

\section{Measures of treatment effect}

For dichotomous outcomes, the risk ratio (RR) will be calculated with $95 \%$ confidence intervals (CIs). For continuous outcome data, we will use the mean difference (MD) with $95 \%$ CIs for trials that use the same assessment scale. When trials use different assessment scales, we will use the standardised mean difference (SMD) with 95\% CIs. Time-to-event data (e.g. time-to-complete wound healing) will be reported as hazard ratios (HRs) when possible, in accordance with the methods described in the Cochrane Handbook for Systematic Reviews of Interventions (Deeks 2011). If studies reporting time-to-event data (e.g. time to healing) do not report a hazard ratio, then, when feasible, we plan to estimate this using other reported outcomes, such as numbers of events, through the application of available statistical methods (Tierney 2007).

\section{Unit of analysis issues}

Unit of analysis issues may arise with studies that include participants with multiple wounds who are treated with the same intervention and report outcomes for each wound, or with studies in which multiple assessments of an outcome are presented for participants. We will record whether trials presented outcomes in relation to a wound, a limb (e.g. foot, leg) or a participant, or as multiple wounds on the same participant. For wound healing, unless otherwise stated, when the number of wounds appears to equal the number of participants, we will treat the wound as the unit of analysis.

When a cluster trial has been conducted and correctly analysed, effect estimates and their standard errors may be meta-analysed using the generic inverse variance method in Review Manager (RevMan 2012). We will also record occasions when multiple wounds on a participant are (incorrectly) treated in the included study as though they are independent of each other, rather than with application of within-patient analysis methods. This will be recorded as part of the 'Risk of bias' assessment.

When a cluster-randomised trial has been conducted but incorrectly analysed at the individual rather than the cluster level, we will approximate the correct analyses if possible, in accordance with Chapter 16 of the Cochrane Handbook for Systematic Reviews of Interventions, using information provided in Higgins 2011b.

- Number of clusters (or groups) randomly assigned to each intervention group; or the average (mean) size of each cluster.

- Outcome data ignoring the cluster design for the total number of individuals (e.g. number or proportion of individuals with events, means and standard deviations).

- Estimate of the intracluster (or intraclass) correlation coefficient (ICC).

\section{Dealing with missing data}

It is common to have data missing from trial reports. Excluding participants from the analysis post randomisation or ignoring participants who are lost to follow-up compromises the randomisation and potentially introduces bias into the trial. If it is thought that study authors might be able to provide some missing data, we will contact them; however, it is likely that data will often be missing because of loss to follow-up. In individual studies, when data on the proportion of ulcers healed are presented, we plan to assume that randomly assigned participants not included in an analysis had an unhealed wound at the end of the follow-up period (i.e. they will be considered in the denominator but not in the numerator). When a trial does not specify participant group numbers before dropout, we will present only complete case data. For time-to-healing analysis using survival analysis methods, dropouts should be accounted for as censored data. Hence all participants will be contributing to the analysis. We acknowledge that such analysis assumes that dropouts are missing at random. We will present data for area change of ulcer and for all secondary outcomes as a complete case analysis.

\section{Assessment of heterogeneity}

We will consider clinical heterogeneity (i.e. the degree to which RCTs vary in terms of participant, intervention and outcome characteristics) and statistical heterogeneity. We will assess statistical 
heterogeneity using the $\mathrm{Chi}^{2}$ test (a significance level of $\mathrm{P}$ less than 0.10 will be considered to indicate statistically significant heterogeneity) in conjunction with the $\mathrm{I}^{2}$ measure (Higgins 2003). $\mathrm{I}^{2}$ examines the percentage of total variation across RCTs that is due to heterogeneity rather than to chance (Higgins 2003). We will consider that $\mathrm{I}^{2}$ values of $40 \%$ or less indicate a low level of heterogeneity, and values of $75 \%$ or more indicate very high heterogeneity (Higgins 2011c).

\section{Assessment of reporting biases}

Reporting biases arise when dissemination of research findings is influenced by the nature and direction of results. Publication bias is one of a number of possible causes of 'small-study effects,' that is, a tendency for estimates of the intervention effect to be more beneficial in smaller RCTs. Funnel plots allow a visual assessment of whether small-study effects may be present in a meta-analysis. A funnel plot is a simple scatter plot of the intervention effect estimates from individual RCTs against some measure of the size or precision of each trial (Sterne 2011). We plan to present funnel plots for meta-analyses comprising 10 or more RCTs using RevMan 5.2.

\section{Data synthesis}

Details of included studies will be combined in a narrative review according to comparators. In terms of meta-analytical approach, in the presence of clinical heterogeneity (review author judgement) and/or evidence of statistical heterogeneity we will use the random-effects model. We will only use a fixed-effect approach when clinical heterogeneity is thought to be minimal and statistical heterogeneity is estimated as non-statistically significant for the ChiSquared value and $0 \%$ for the $\mathrm{I}^{2}$ assessment (Kontopantelis 2012). For dichotomous outcomes, we will present the summary estimate as a risk ratio (RR) with $95 \%$ CI. When continuous outcomes are measured in the same way across studies, we plan to present a pooled mean difference (MD) with $95 \%$ CI. We plan to pool standardised mean difference (SMD) estimates when studies have measured the same outcome using different methods. Pooled data will be presented using forest plots. For time-to-event data, we plan to plot (and, if appropriate, pool) estimates of HRs and 95\% CIs as presented in the study reports using the generic inverse variance method in RevMan 5.2. Pooled estimates of treatment effect will be obtained by using RevMan (RevMan 2012).

\section{'Summary of findings' tables}

We will present the main results of the review in 'Summary of findings' tables. These tables present key information concerning the quality of the evidence, the magnitude of the effects of the interventions examined and the sum of available data for the main outcomes (Schunemann 2011a). The 'Summary of findings' tables also include an overall grading of the evidence related to each of the main outcomes using the GRADE (Grades of Recommendation, Assessment, Development and Evaluation) approach. The GRADE approach defines the quality of a body of evidence as the extent to which one can be confident that an estimate of effect or association is close to the true quantity of specific interest. The quality of a body of evidence involves consideration of withintrial risk of bias (methodological quality), directness of evidence, heterogeneity, precision of effect estimates and risk of publication bias (Schunemann 2011b). We plan to present the following outcomes in the 'Summary of findings' tables.

- Time to complete ulcer healing when analysed using appropriate survival analysis methods.

- Proportion of ulcers completely healing during the trial period.

- Adverse events.

\section{Subgroup analysis and investigation of heterogeneity}

When possible, we will perform a subgroup analysis to explore the influence of the following factor on effect sizes.

- Ulcer category: When possible, we will assess whether there are differences in effect sizes for grade 2 pressure ulcers and for the more severe grade 3 and 4 pressure ulcers.

\section{Sensitivity analysis}

When possible, we will perform sensitivity analyses to explore the influence of the following factor on effect sizes.

- Risk of bias: We will assess the influence of removing from meta-analyses studies classed as having high and unclear risk of bias. We will include only studies that are assessed as having low risk of bias in all key domains, namely, adequate generation of the randomisation sequence, adequate allocation concealment and blinding of outcome assessor, for the estimates of treatment effect.

\section{ACKNOWLEDGEMENTS}

The review authors are grateful to the following peer reviewers for their time and comments: Elizabeth McInnes, Gill Norman, Gill Worthy and Zena Moore. The review authors would like to acknowledge the contribution of the copy editor, Dolores Matthews.

This report describes independent research funded by the National Institute for Health Research (NIHR Cochrane Programme Grant 13/89/08-High Priority Cochrane Reviews in Wound Prevention and Treatment). The views expressed in this publication are those of the review author(s) and are not necessarily those of the NHS, the National Institute for Health Research or the Department of Health. 


\section{R E F E R E N C E S}

\section{Additional references}

\section{Allman 1997}

Allman RM. Pressure ulcer prevalence, incidence, risk factors, and impact. Clinical Geriatric Medicine 1997;13(3): 421-36.

Allman 1999

Allman RM, Goode PS, Burst N, Bartolucci AA, Thomas DR. Pressure ulcers, hospital complications, and disease severity: impact on hospital costs and length of stay. Advances in Wound Care 1999;12:22-30.

\section{AWMA 2012}

Australian Wound Management Association and Pan Pacific partners. Pan Pacific clinical practice guideline for the prevention and management of pressure injury. Cambridge Publishing on-line report. Perth WA: Cambridge Publishing, 2012:http://www.awma.com.au/publications/ 2012_AWMA_Pan_Pacific_Guidelines.pdf (access 6th June 2014).

\section{Bennett 2004}

Bennett G, Dealey C, Posnett J. The cost of pressure ulcers in the UK. Age and Ageing 2004;33(3):230-5.

\section{Bergstrom 1998}

Bergstrom N, Braden B, Champagne M, Kemp M, Ruby E. Predicting pressure ulcer risk: a multisite study of the predictive validity of the Braden Scale. Nursing Research 1998;47(5):261-9.

\section{Berlowitz 1990}

Berlowitz DR, Wilikin SVB. Risk factors for pressure sores: a comparison of cross-sectional and cohort derived data. Journal of the American Geriatrics Society 1990;37(11): 1043-50.

\section{Berlowitz 1997}

Berlowitz DR, Brandeis GH, Anderson J, Brand HK. Predictors of pressure ulcer healing among long-term care residents. Journal of the American Geriatrics Society 1997;45 (1):30-4.

BNF 2013

British National Formulary, British Medical Association, British Royal Pharmaceutical Society of Great Britain. British National Formulary 66. London: British Medical Association, 2013

\section{Brandeis 1994}

Brandeis GH, Ooi WL, Hossain M, Morris JN, Lipsitz LA. A longitudinal study of risk factors associated with the formation of pressure ulcers in nursing homes. Journal of the American Geriatric Society 1994;42:388-93.

\section{Cardinal 2009}

Cardinal M, Eisenbud DE, Armstrong DG, Zelen C Driver V, Attinger C, et al.Serial surgical debridement: a retrospective study on clinical outcomes in chronic lower extremity wounds. Wound Repair and Regeneration 2009;17 (3):306-11.
Dealey 2012

Dealey C, Posnett J, Walker A. The cost of pressure ulcers in the United Kingdom. Journal of Wound Care 2012;21: 261-2, 264, 266.

Deeks 2011

Deeks JJ, Higgins JPT, Altman DG (editors). Chapter 9: Analysing data and undertaking meta-analyses. In: Higgins JPT, Green S (editors). Cochrane Handbook for Systematic Reviews of Interventions. 5.1.0 (updated March 2011) edition. The Cochrane Collaboration, 2011. www.cochrane-handbook.org.

Donini 2005

Donini LM, De Felice MR, Tagliaccica A, De Bernardini L, Cannella C. Nutritional status and evolution of pressure sores in geriatric patients. Journal Nutrition and Health in Aging 2005;9:446-54.

\section{EPUAP-NPUAP 2009}

European Pressure Ulcer Advisory Panel and National Pressure Ulcer Advisory Panel. Pressure ulcer treatment: quick reference guide. Washington DC: National Pressure Ulcer Advisory Panel, 2009.

\section{Essex 2009}

Essex HN, Clark M, Sims J, Warriner A, Cullum N. Health-related quality of life in hospital inpatients with pressure ulceration: assessment using generic health-related quality of life measures. Wound Repair Regeneration 2009; 17:797-805.

Gebhardt 2002

Gebhardt K. Pressure ulcer prevention. Part 1. Causes of pressure ulcers. Nursing Times 2002;98:41-4.

\section{Gefen 2014}

Gefen A. Tissue changes in patients following spinal cord injury and implications for wheelchair cushions and tissue loading: a literature review. Ostomy Wound Management 2014;60:34-45.

\section{Graves 2005}

Graves N, Birrell F, Whitby M. Modelling the economic losses from pressure ulcers among hospitalized patients in Australia. Wound Repair and Regeneration 2005;13:462-7.

Higgins 2003

Higgins JPT, Thompson SG, Deeks JJ, Altman DG. Measuring inconsistency in meta-analyses. BMJ 2003;327 (7414):557-60

Higgins 2011a

Higgins JPT, Altman DG, Sterne, JAC (editors). Chapter 8: Assessing risk of bias in included studies. In: Higgins JPT, Green S (editors). Cochrane Handbook for Systematic Reviews of Interventions Version 5.1.0 [updated March 2011]. The Cochrane Collaboration, 2011. www.cochranehandbook.org.

\section{Higgins 2011b}

Higgins JPT, Deeks JJ, Altman DG (editors). Chapter 16: Special topics in statistics. In: Higgins JPT, Green $S$ 
(editors). Cochrane Handbook for Systematic Reviews of Interventions. Version 5.1.0 [updated March 2011]. The Cochrane Collaboration, 2011. www.cochranehandbook.org.

Higgins 2011c

Higgins JPT, Deeks JJ. Chapter 7: Selecting studies and collecting data. In: Higgins JPT, Green $S$ (editors). Cochrane Handbook for Systematic Reviews of Interventions Version 5.1.0 [updated March 2011]. The Cochrane Collaboration, 2011. www.cochranehandbook.org.

\section{Kontopantelis 2012}

Kontopantelis E, Springate DA, Reeves D. A re-analysis of the Cochrane Library data: the dangers of unobserved heterogeneity in meta-analyses.. PLoS One 2013;26: e69930.

\section{Langer 2003}

Langer G, Knerr A, Kuss O, Behrens J, Schlömer GJ. Nutritional interventions for preventing and treating pressure ulcers. Cochrane Database of Systematic Reviews 2003, Issue 4. [DOI: 10.1002/14651858.CD003216]

\section{Lefebvre 2011}

Lefebvre C, Manheimer E, Glanville J. Chapter 6: Searching for studies. In: Higgins JPT, Green S (editors). Cochrane Handbook for Systematic Reviews of Interventions. 5.1.0 [updated March 2011]. The Cochrane Collaboration, 2011. www.cochrane-handbook.org.

\section{Liberati 2009}

Liberati A, Altman DG, Tetzlaff J, Mulrow C, Gotzsche PC, Ioannidis JP, et al.The PRISMA statement for reporting systematic reviews and meta-analyses of studies that evaluate health care interventions: explanation and elaboration. PLoS Medicine 2009;6:e1000100.

\section{McInnes 2011}

McInnes E, Dumville JC, Jammali-Blasi A, Bell-Syer SE. Support surfaces for treating pressure ulcers. Cochrane Database of Systematic Reviews 2011, Issue 12. [DOI: 10.1002/14651858.CD009490]

National Safety Thermometer Data 2014

National Health Service. National Safety Thermometer Data. http://www.safetythermometer.nhs.uk/index.php? option=com_dashboards\&view=classic\&Itemid=126 2014: (accessed 4 March 2014).

\section{NPUAP 2009}

The National Pressure Ulcer Advisory Panel. NPUAP Pressure Ulcer Stages/Categories. http://www.npuap.org/ resources/educational-and-clinical-resources/npuappressure-ulcer-stagescategories/ (accessed 4 March 2014).

Pieper 1995

Pieper B, Mott M. Nurses' knowledge of pressure ulcer prevention, staging, and description. Advances in Wound Care 1995;8(3):34, 38, 40.

Power 2012

Power M, Stewart K, Brotherton A. What is the NHS Safety Thermometer?. Clinical Risk 2012;18:163-9.
Reddy 2008

Reddy M, Gill SS, Kalkar SR, Wu W, Anderson PJ, Rochon PA. Treatment of pressure ulcers: a systematic review. Journal of the American Medical Association 2008;300: 2647-62.

RevMan 2012

The Nordic Cochrane Centre. Review Manager (RevMan) 5.2. The Cochrane Collaboration, 2012.

\section{Russo 2008}

Russo A, Steiner C, Spector W. Hospitalizations related to pressure ulcers among adults 18 years and older, 2006. Healthcare Cost and Utilization Project (HCUP). Acessed 6th June 2014 2008:http://www.hcup-us.ahrq.gov/reports/ statbriefs/sb64.jsp (accessed 6 June 2014).

\section{Schunemann 2011a}

Schünemann HJ, Oxman AD, Higgins JPT, Vist GE, Glasziou P, Guyatt GH. Chapter 11: Presenting results and 'Summary of findings' tables. In: Higgins JPT, Green $S$ (editors). Cochrane Handbook for Systematic Reviews of Interventions Version 5.1.0 [updated March 2011]. The Cochrane Collaboration, 2011. www.cochranehandbook.org.

\section{Schunemann 2011b}

Schünemann HJ, Oxman AD, Higgins JPT, Deeks JJ, Glasziou P, Guyatt GH. Chapter 12: Interpreting results and drawing conclusions. In: Higgins JPT, Green S (editors). Cochrane Handbook for Systematic Reviews of Interventions Version 5.1.0 [updated March 2011]. The Cochrane Collaboration, 2011. www.cochranehandbook.org.

\section{SIGN 2011}

Scottish Intercollegiate Guidelines Network (SIGN). Search filters. http://www.sign.ac.uk/methodology/filters.html\# random. (accessed December 2011).

\section{Smith 2013}

Smith ME, Totten A, Hickam DH, Fu R, Wasson N, Rahman B, et al.Pressure ulcer treatment strategies: a systematic comparative effectiveness review. Annals of Internal Medicine 2013;159:39-50.

\section{Sterne 2011}

Sterne JAC, Egger M, Moher D. Chapter 10: Addressing reporting biases. In: Higgins JPT, Green S (editors). Cochrane Handbook for Systematic Reviews of Interventions Version 5.1.0 [updated March 2011]. The Cochrane Collaboration, 2011. www.cochranehandbook.org.

\section{Stevenson 2013}

Stevenson R, Collinson M, Henderson V, Wilson L, Dealey $\mathrm{C}, \mathrm{McGinnis} \mathrm{E}$, et al.The prevalence of pressure ulcers in community settings: an observational study. International Journal of Nursing Studies 2013;50:1550-7.

\section{Tierney 2007}

Tierney JF, Stewart LA, Ghersi D, Burdett S, Sydes MR. Practical methods for incorporating summary time-to-event data into meta-analysis. Trials 2007;7(8):16. 
Vanderwee 2007

Vanderwee K, Clark M, Dealey C, Gunningberg L, Defloor

T. Pressure ulcer prevalence in Europe: a pilot study. Journal

of Evaluation in Clinical Practice 2007;13(2):227-35.

VanGilder 2009

VanGilder C, Amlung S, Harrison P, Meyer S. Results of the 2008-2009 International Pressure Ulcer Prevalence Survey and a 3-year, acute care, unit-specific analysis. Ostomy/ Wound Management 2009;55(11):39-45.

Winter 1962

Winter GD. Formation of the scab and the rate of epithelization of superficial wounds in the skin of the young

domestic pig. Nature 1962;193:293-4.

\section{Winter 1963a}

Winter GD, Scales JT. Effect of air drying and dressings on the surface of a wound. Nature 1963;197:91-2.

\section{Winter $1963 b$}

Winter GD. Effect of air exposure and occlusion on experimental human skin wounds. Nature 1963;200: 378-9.

* Indicates the major publication for the study

\section{A P P E N D I C ES}

\section{Appendix I. Risk of bias assessment (individually randomised controlled trials)}

1. Was the allocation sequence randomly generated?

Low risk of bias:

The investigators describe a random component in the sequence generation process, such as referring to a random number table; using a computer random number generator; tossing a coin; shuffling cards or envelopes; throwing dice; drawing of lots.

High risk of bias:

The investigators describe a non-random component in the sequence generation process. Usually, the description would involve some systematic, non-random approach, for example, sequence generated by odd or even date of birth; sequence generated by some rule based on date (or day) of admission; sequence generated by some rule based on hospital or clinic record number.

Unclear:

Insufficient information about the sequence generation process to permit judgement of low or high risk of bias.

2. Was the treatment allocation adequately concealed?

Low risk of bias:

Participants and investigators enrolling participants could not foresee assignment because one of the following, or an equivalent method, was used to conceal allocation: central allocation (including telephone, web-based and pharmacy-controlled randomisation); sequentially numbered drug containers of identical appearance; sequentially numbered, opaque, sealed envelopes.

High risk of bias:

Participants or investigators enrolling participants could possibly foresee assignments and thus introduce selection bias, such as allocation based on using an open random allocation schedule (e.g. a list of random numbers); assignment envelopes were used without appropriate safeguards (e.g. if envelopes were unsealed or non-opaque or were not sequentially numbered); alternation or rotation; date of birth; case record number; any other explicitly unconcealed procedure.

Unclear:

Insufficient information to permit judgement of low or high risk of bias. This is usually the case if the method of concealment is not described or is not described in sufficient detail to allow a definitive judgement, for example, if the use of assignment envelopes is described, but it remains unclear whether envelopes were sequentially numbered, opaque and sealed.

3. Blinding (Participants, personnel and outcome assessors) - was knowledge of the allocated interventions adequately prevented during the study?

Low risk of bias:

Any one of the following:

- No blinding, but the review authors judge that the outcome and the outcome measurement are not likely to be influenced by lack of blinding.

- Blinding of participants and key study personnel ensured, and unlikely that the blinding could have been broken. 
- Either participants or some key study personnel were not blinded, but outcome assessment was blinded and the non-blinding of others is unlikely to introduce bias.

High risk of bias:

Any one of the following:

- No blinding or incomplete blinding, and the outcome or outcome measurement is likely to be influenced by lack of blinding.

- Blinding of key study participants and personnel attempted, but likely that the blinding could have been broken.

- Either participants or some key study personnel were not blinded, and the non-blinding of others is likely to introduce bias.

Unclear:

Any one of the following:

- Insufficient information to permit judgement of low or high risk of bias.

- The study did not address this outcome.

4. Were incomplete outcome data adequately addressed?

Low risk of bias:

Any one of the following:

- No missing outcome data.

- Reasons for missing outcome data unlikely to be related to true outcome (for survival data, censoring unlikely to be introducing bias).

- Missing outcome data balanced in numbers across intervention groups, with similar reasons for missing data across groups.

- For dichotomous outcome data, the proportion of missing outcomes compared with observed event risk not enough to have a clinically relevant impact on the intervention effect estimate.

- For continuous outcome data, plausible effect size (difference in means or standardised difference in means) among missing outcomes not enough to have a clinically relevant impact on observed effect size.

- Missing data have been imputed using appropriate methods.

High risk of bias:

Any one of the following:

- Reason for missing outcome data likely to be related to true outcome, with imbalance in numbers or reasons for missing data across intervention groups.

- For dichotomous outcome data, the proportion of missing outcomes compared with observed event risk enough to induce clinically relevant bias in intervention effect estimate.

- For continuous outcome data, plausible effect size (difference in means or standardised difference in means) among missing outcomes enough to induce clinically relevant bias in observed effect size.

- 'As-treated' analysis done with substantial departure of the intervention received from that assigned at randomisation.

- Potentially inappropriate application of simple imputation.

Unclear:

Any one of the following:

- Insufficient reporting of attrition/exclusions to permit judgement of low or high risk of bias (e.g. number randomised not stated, no reasons for missing data provided).

- The study did not address this outcome.

5. Are reports of the study free of the suggestion of selective outcome reporting?

Low risk of bias:

Any one of the following:

- The study protocol is available and all of the study's prespecified (primary and secondary) outcomes that are of interest in the review have been reported in the prespecified way.

- The study protocol is not available but it is clear that the published reports include all expected outcomes, including those that were prespecified (convincing text of this nature may be uncommon).

High risk of bias:

Any one of the following:

- Not all of the study's prespecified primary outcomes have been reported.

- One or more primary outcomes are reported using measurements, analysis methods or subsets of the data (e.g. subscales) that were not prespecified.

Alginate dressings for treating pressure ulcers (Protocol)

Copyright (c) 2014 The Cochrane Collaboration. Published by John Wiley \& Sons, Ltd. 
- One or more reported primary outcomes were not prespecified (unless clear justification for their reporting is provided, such as an unexpected adverse effect).

- One or more outcomes of interest in the review are reported incompletely so that they cannot be entered in a meta-analysis.

- The study report fails to include results for a key outcome that would be expected to have been reported for such a study.

Unclear:

Insufficient information to permit judgement of low or high risk of bias. It is likely that the majority of studies will fall into this category.

6. Other sources of potential bias

Low risk of bias:

The study appears to be free of other sources of bias.

High risk of bias:

There is at least one important risk of bias. For example, the study:

- had a potential source of bias related to the specific study design used; or

- has been claimed to have been fraudulent; or

- had some other problem.

Unclear:

There may be a risk of bias, but there is either:

- insufficient information to assess whether an important risk of bias exists; or

- insufficient rationale or evidence that an identified problem will introduce bias.

\section{Appendix 2. Risk of bias assessment (cluster randomised controlled trials)}

In cluster randomised trials, particular biases to consider include recruitment bias; baseline imbalance; loss of clusters; incorrect analysis; and comparability with individually randomised trials.

- Recruitment bias can occur when individuals are recruited to the trial after the clusters have been randomly assigned, as knowledge of whether each cluster is an 'intervention' or 'control' cluster could affect the types of participants recruited.

- Cluster randomised trials often randomly assigned all clusters at once, so lack of concealment of an allocation sequence should not usually be an issue. However, because small numbers of clusters are randomly assigned, there is a possibility of chance baseline imbalance between randomly assigned groups, in terms of the clusters or the individuals. Although not a form of bias as such, the risk of baseline differences can be reduced by using stratified or pair-matched randomisation of clusters. Reporting of the baseline comparability of clusters, or statistical adjustment for baseline characteristics, can help reduce concern about the effects of baseline imbalance.

- Occasionally, complete clusters are lost from a trial and have to be omitted from the analysis. Just as for missing outcome data in individually randomised trials, this may lead to bias. In addition, missing outcomes for individuals within clusters may lead to risk of bias in cluster randomised trials.

- Many cluster randomised trials are analysed by incorrect statistical methods, without taking the clustering into account. Such analyses create a 'unit of analysis error' and produce overly precise results (the standard error of the estimated intervention effect is too small) and P values that are too small. They do not lead to biased estimates of effect. However, if they remain uncorrected, they will receive too much weight in a meta-analysis.

- In a meta-analysis including both cluster and individually randomised trials, or including cluster randomised trials with different types of clusters, possible differences between the intervention effects estimated need to be considered. For example, in a vaccine trial of infectious diseases, a vaccine applied to all individuals in a community would be expected to be more effective than vaccine applied to only half of the people. Another example is provided by a Cochrane review of hip protectors. The cluster trials showed a large positive effect, whereas individually randomised trials did not show clear benefit. One possibility is that there was a 'herd effect' in the cluster randomised trials (which were often performed in nursing homes, where compliance with using the protectors may have been enhanced). In general, such 'contamination' would lead to underestimates of effect. Thus, if an intervention effect is still demonstrated despite contamination in those trials that were not cluster randomised, a confident conclusion about the presence of an effect can be drawn. However, the size of the effect is likely to be underestimated. Contamination and 'herd effects' may be different for different types of clusters.

Alginate dressings for treating pressure ulcers (Protocol) 


\section{CONTRIBUTIONSOFAUTHORS}

Jo Dumville developed the protocol and co-ordinated its development, completed the first draft of the protocol, co-ordinated edits of subsequent drafts, made an intellectual contribution, approved the final version before submission and is the guarantor of the protocol.

Nikki Stubbs completed the first draft of the protocol and made an intellectual contribution to and approved the final version of the protocol before submission.

Samantha Keogh completed the first draft of the protocol and made an intellectual contribution to and approved the final version of the protocol before submission.

Rachel Walker completed the first draft of the protocol and made an intellectual contribution to and approved the final version of the protocol before submission.

Matthew Fortnam commented on the protocol draft and approved the final version of the protocol before submission.

\section{Contributions of editorial base}

Nicky Cullum: advised on methodology, interpretation and content; and edited the protocol and approved the protocol for submission. Sally Bell-Syer: co-ordinated the editorial process; advised on methodology, interpretation and content; and edited the protocol. Amanda Briant: designed the search strategy and edited the search methods section.

\section{DECLARATIONSOF INTEREST}

Jo C Dumville: nothing to declare.

Samantha Keogh: nothing to declare.

Nikki Stubbs has received funding from Pharmaceutical companies to support training and education events in the service and payments have been received by the author for non product related educational sessions. These have been unrelated to the subject matter of the systematic review and have never been in support or in pursuit of the promotion of products.

Rachel Walker: is currently employed by the National Health and Medical Research Council's Centre of Research Excellence in Nursing (NCREN), Griffith University Australia. Skin integrity including pressure ulcers is a research foci of NCREN.

Matthew Fortnam: nothing to declare.

This protocol/review is part of a suite of reviews investigating the use of individual dressing types in the treatment of pressure ulcers. The first review in this suite was developed by co-author Samantha Keogh. The objective of undertaking these reviews is to use them in an overview which draws together all existing Cochrane review evidence regarding the use of dressing treatments for pressure ulcers to guide clinician decision-making.

\section{SOURCES OF SUPPORT}




\section{Internal sources}

- The National Institute for Health Research (NIHR) is the sole funder of the Cochrane Wounds Review Group, UK.

- School of Nursing, Midwifery and Social Work. University of Manchester, UK.

\section{External sources}

- NIHR Cochrane Programme Grant Project: 13/89/08-High Priority Cochrane Reviews in Wound Prevention and Treatment, UK. 